\title{
Simulation du comportement d'une galerie de mine et de son soutènement lors d'un phénomène de rupture brutale
}

\section{G. COLOMBET}

Coyne et Bellier

9, allée des Barbanniers 92632 Gennevilliers cedex

\section{F. ESTEULLE}

Coyne et Bellier clo Europact Ltda. Orrego Luco 87.

$6^{\circ}$ Piso, of 5

Santiago (Chili)

Les ruptures brutales, ou $\alpha$ coups de toit », ou rockbursts sont des phénomènes redoutés dans les mines profondes, à cause de leur violence et de leur soudaineté. Les dommages qu'ils causent aux galeries posent, en effet, de graves problèmes de sécurité. La fréquence de ces événements s'étant récemment accrue dans les niveaux les plus profonds de la mine d'El Teniente (Chili), l'objet. de cette étude a été d'examiner, à l'échelle d'une galerie de mine, l'influence de ces phênomènes et le rôle des éléments de soutènement des galeries.

Un modèle numérique bidimensionnel permettant la simulation de l'excavation et de la mise en place du soutènement d'une galerie, puis de la progression de Y'exploitation minière a donc été conçu. Le rockburst a ensuite été simulé par l'application d'un train d'ondes sismiques sur le modèle, en prenant soin de se replacer autant que possible dans les conditions d'un rockburst réellement observé.

Le calcul a été mené avec le code de calcul aux éléments finis GEFDYN.

Le modèle a montré la très forte influence du mode d'exploitation par foudroyage sur la galerie et son soutènement. La simulation du rockburst lui-même a pu être menée de manière satisfaisante, mais a mis en évidence le caractère encore incomplet des données concernant ce type de phénomène.

Des recommandations de soutènement pour

l'amélioration de la tenue des galeries ont néanmoins pu être formulées à l'issue de cette étude.

\section{Simulation of the behaviour of a mine gallery and its support elements during a rockburst}

Rockbursts phenomena occuring in deep mines are feared because of their violence and suddenness. The damages they cause to mine adits pose big security problems. As the frequency of rockburst events recently increased in the deepest level of the El Teniente copper mine (Chile), it was decided to analyse the influence of a rockburst on a mine gallery and its support elements. A bidimensional computer modelling allowing for simulation of the excavation, placement of the support and progressive mining exploitation was achieved. Then the rockburst was simulated by submitting the model to seismic stress waves, taking care to reproduce as well as possible the conditions of one of the rockburst events that really occured

The computer modelling was achieved using the FEM code GEFDYN.

The results evidenced the very strong influence of the caving exploitation on the mine gallery and its support elements. Although the rockburst simulation itself could be successefully achieved, it evidenced a remaining lack of data towards such phenomena.

Recommandations regarding to the improvement of the support. efficiency towards rockbursts could be formulated, too. 


\section{Introduction}

La mine souterraine de cuivre d'El Teniente, au Chili, est située dans les Andes, à une centaine de kilomètres au sud de la capitale, Santiago. Son exploitation industrielle a débuté dès le début du siècle ; elle est actuellement la plus grande mine souterraine de cuivre du monde.

Avec l'épuisement des niveaux supérieurs, l'exploitation s'est étendue en profondeur, jusque dans la roche dite primaire, plus dure et plus compacte que la roche dite secondaire, plus décomprimée, dans laquelle l'exploitation se situait jusqu'alors.

Il y a environ cinq ans a été ouvert le niveau Sub 6, à une altitude moyenne de $2100 \mathrm{~m}$, soit à environ $1000 \mathrm{~m}$ de profondeur (la surface du sol au-dessus de la mine se situe à une altitude variant entre $2900 \mathrm{~m}$ et
$3200 \mathrm{~m}$ d'altitude). Ce niveau est actuellement le plus profond de la mine.

Dès l'ouverture du niveau Sub 6, des rockbursts (ou encore ( coups de toit 》) ont commencé à s'y produire à une fréquence anormalement élevée. Ces rockbursts, parfois très violents, ont provoqué l'endommagement et l'effondrement de plusieurs galeries. Leur caractère imprévisible, sans relation avec l'activité normale de la mine (tirs, etc.) a rapidement posé aux responsables de la mine de graves problèmes de sécurité des personnes, à tel point que I'exploitation du niveau Sub 6 a dû être arrêtée et abandonnée provisoirement.

L'objectif de l'étude a donc été l'analyse du comportement d'une galerie de mine et de ses éléments de soutènement pendant les différentes phases de l'exploitation et sous l'effet des sollicitations dynamiques provoquées par un phénomène de rockburst. Cette étude devait en outre permettre d'analyser plus précisément le rôle des différents éléments de soutènement et la manière dont ce soutènement pourrait éventuellement être aclapté pour faire face à ces phénomènes.

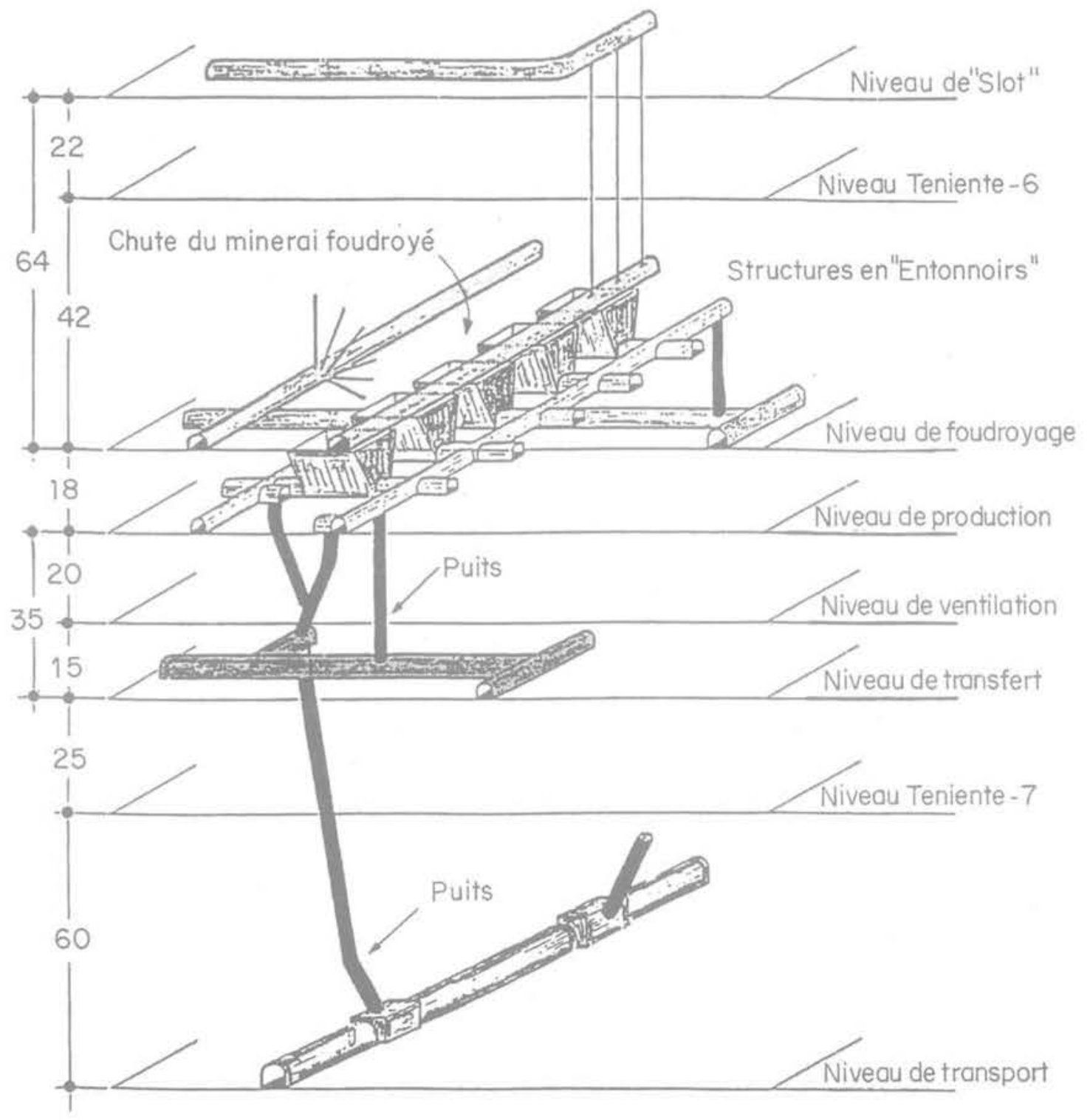

FG.1 Système d'exploitation par foudroyage en panel caving. Exploitation by means of panel caving. 


\section{Le mode d'exploitation de la mine et ses conséquences sur le massif rocheux}

Afin d'essayer de mieux comprendre la nature et la cause des phénomènes se produisant au sein du massif rocheux à la mine d'El Teniente, il est nécessaire de décrire la méthode d'exploitation par foudroyage utilisée dans la mine.

Le cuivre étant disséminé dans la masse de la roche, le minerai est constitué par les roches encaissantes elles-mêmes.

Au niveau Sub 6, le système d'exploitation est un système de foudroyage par panneaux, ou panel caving, illustré par la figure 1. Plusieurs niveaux de galeries sont excavés, le principal étant le niveau de production, constitué d'un quadrillage de galeries en voûte desquelles sont excavées, à intervalles réguliers, de grandes structures en entonnoirs ouvertes vers le haut.

Le massif rocheux sus-jacent est prédécoupé sur une hauteur d'environ $70 \mathrm{~m}$. Puis, à partir de forages exécutés depuis les galeries du niveau de foudroyage, des tirs sont effectués, de manière que le minerai tombe par gravité dans les structures en entonnoirs préalablement excavées, à la base desquelles le minerai est chargé et évacué gravitairement par puits jusqu'aux niveaux de transport inférieurs (voir figure 1).

Le principe du mode d'exploitation est similaire dans les autres niveaux d'exploitation de la mine.

Un tel mode d'exploitation par foudroyage, puis soutirage de portions considérables du massif rocheux n'est évidemment pas sans conséquences. C'est ainsi que l'état des contraintes géostatiques au sein du massif rocheux va se trouver considérablement modifié par la présence, après foudroyage, de zones « foisonnées » de très grand volume, autour desquelles les contraintes vont devoir se réorganiser.

C'est donc finalement le champ des contraintes géostatiques à l'échelle de tout le massif rocheux qui subit cle profondes modifications avec la progression de l'exploitation dans la mine. Le champ des contraintes autour des galeries se trouve donc lui-même profondément affecté par les différentes phases de l'exploitation et, en particulier, le foudroyage.

Il faut, dès lors, s'attendre à ce que le massif rocheux, au voisinage des zones foudroyées, se réajuste spontanément pour s'adapter au nouvel état de contraintes. C'est très probablement dans ce réajustement, qui s'opère vraisemblablement par mouvements le long de discontinuités géologiques existantes, qu'il faut chercher la cause des phénomènes de rockbursts observés.

Il est, par exemple, remarquable de constater que lors du rockburst du 23 mai 1991, qui a très gravement endommagé plusieurs galeries du niveau Sub 6 de la mine, les sismographes du pays ont enregistré une secousse sismique de magnitude 3 dont l'épicentre s'est trouvé situé... à la mine d'El Teniente !

Ceci a montré que les phénomènes de rockbursts étaient bien accompagnés de phénomènes sismiques, dont la magnitude pouvait être estimée.

Les variations de l'état des contraintes géostatiques apparaissent donc essentielles pour la modélisation des rockbursts. Il faudra, en particulier, tenir compte du fait que les rockbursts surviennent souvent environ $100 \mathrm{~m}$ en avant du front (limite de la zone foudroyée, qui progresse avec l'exploitation), ou autour des zones foudroyées.

\section{3}

\section{Élaboration du modèle numérique}

Dans le cadre de la modélisation par la méthode des éléments finis, on a cherché autant que possible à se replacer dans les conditions d'un rockburst réellement observé, en l'occurrence celui du 23 mai 1991, dont la magnitude était connue (voir ci-dessus).

Connaissant la localisation des diverses galeries endommagées lors de cet événement, il a été décidé de modéliser une des galeries endommagées au niveau de ventilation, plutôt qu'une galerie du niveau de production, où l'effet tridimensionnel est très marqué, et se prête donc mal à la représentation par un modèle bidimensionnel (voir figure 1).

La figure 2 représente le maillage utilisé pour la présente étude. Il représente un bloc de massif rocheux de $55 \mathrm{~m}$ de haut pour $30 \mathrm{~m}$ de large. Pour la partie statique de la modélisation, nous avons tenu compte de la présence d'autres galeries au-dessus et au-dessous du niveau de ventilation (niveau de production et niveau de transport).

Ce modèle inclut les éléments de soutènement de la galerie (ancrages à scellement continu et béton projeté armé de treillis soudé), suivant les modalités qui seront développées ci-dessous.

\section{Données et hypothèses de calcul}

\section{1}

\section{Choix des données pour la modélisation}

Les résultats obtenus par un calcul aux éléments finis dépendant entièrement des données qui y sont introduites, une grande attention a été prêtée à la détermination des divers paramètres à introduire dans le modèle.

Ces paramètres étaient d'une part, ceux relatifs à la description des caractéristiques mécaniques du massif rocheux et des éléments de soutènement et, d'autre part, la variation des champs de contraintes statiques (avec les phases d'exploitation) et dynamiques (sollicitations sismiques lors d'un rockburst).

Nous allons détailler dans les paragraphes qui suivent les différentes hypothèses adoptées pour la modélisation.

\section{2}

\section{Caractéristiques du massif rocheux}

La roche au niveau Sub 6 de la mine d'El Teniente est essentiellement constituée d'andésites et de petits corps intrusifs de diorite. 


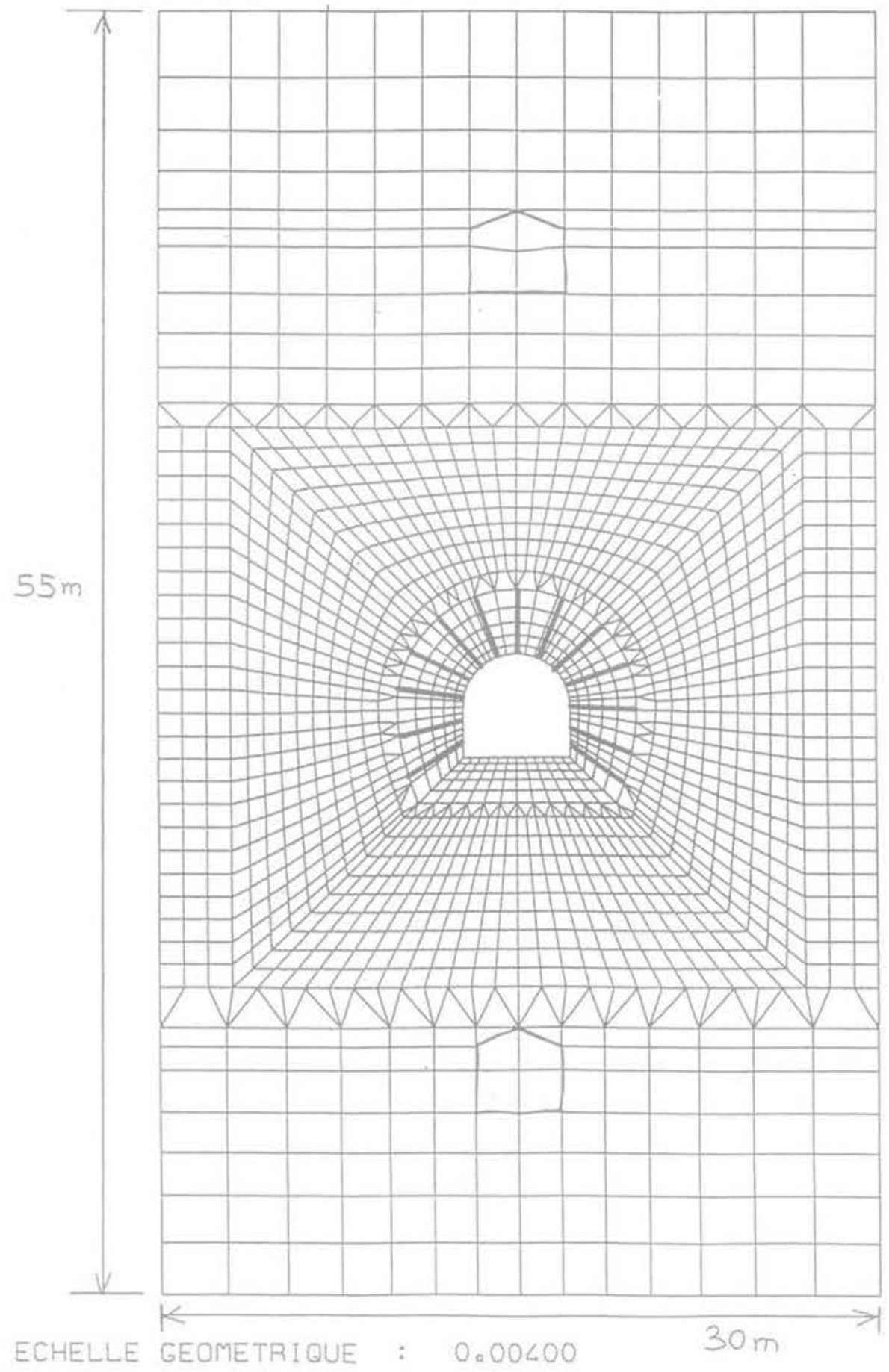

FG. 2: Maillage utilisé dans la modélisation; les traits gras figurent les ancrages à scellement continu. Modiel mesh used for the simulation; thick strokes show the position of the grouted anchors.

L'estimation des caractéristiques mécaniques du massif rocheux s'est faite surtout sur la base des observations faites dans la mine, ainsi que sur l'expérience du personnel spécialisé de la mine, habitué à décrire les différentes formations rocheuses rencontrées par le système du rock mass rating (désigné par RMR dans la suite) de la classification de Bieniawski.

Pour la modélisation, les caractéristiques du massif rocheux ont été choisies de manière à représenter la formation rocheuse dite " andésite cisaillée » par les géologues de la mine, de loin la plus représentée au niveau Sub 6.
Il était, toutefois, nécessaire d'estimer, pour la modélisation, le critère de rupture à adopter pour la masse rocheuse (par opposition aux criteres de rupture définis à partir d'essais sur échantillons de petite taille, qui n'incluent donc pas, en particulier, l'effet des discontinuités d'un massif rocheux).

Il a été décidé, en accord avec les géomécaniciens de la mine, que le massif rocheux serait décrit par un critère de rupture du type Hoek-Brown:

$$
\sigma_{1}^{\prime}=\sigma_{3}^{\prime}+\sqrt{m \sigma_{c} s_{3}^{\prime}+s \sigma_{c}^{2}}
$$


où $\sigma_{1}^{\prime}$ et $\sigma_{3}^{\prime}$ sont respectivement les contraintes principales effectives majeure et mineure et $\sigma$ la résistance uniaxale à la compression de la roche intacte.

A partir des très nombreux résultats d'essais en laboratoire disponibles, la valeur moyenne de $110 \mathrm{MPa}$ a été choisie pour $\sigma_{c}$.

Sachant que le RMR attribué à l'andésite cisaillée vaut 62 , les valeurs des paramètres $m$ et $s$ ont été estimées à l'aide des formules recommandées par Hoek et Brown (Hoek, 1988) d'où :

$$
m=4,376 \quad \text { et } \quad s=0,0147
$$

Le critère de rupture obtenu de cette manière représente le comportement du matériau à l'échelle du massif rocheux, la nature et les caractéristiques des discontinuités du massif étant prises en compte par l'intermédiaire du RMR de la classification de Bieniawski.

Les calculs aux éléments finis effectués par la suite l'ont donc été en considérant le matériau comme élastoplastique, le seuil plastique étant défini par le critère de rupture ainsi défini.

Afin de tenir compte du phénomène de radoucissement dans les calculs, le comportement post-rupture du matériau a été simulé par l'adoption, pour les zones ayant atteint le seuil plastique, d'un critère de rupture «radouci », limitant les états de contraintes admissibles dans les zones plastifiées.

Dans ces zones plastifiées, le nouveau critère dé rupture est défini de la même façon que ci-dessus, les paramètres $m$ et $s$ étant déduits de la valeur du RMR attribuée aux zones décomprimées observées dans la mine, égale à 48 . On obtient alors :

$$
m=2,654 \quad \text { et } \quad s=0,0031
$$

Les essais de laboratoire effectués sur éprouvettes ne permettant pas l'estimation de caractéristiques de déformation à l'échelle du massif rocheux, il a été décidé, en l'absence de donnés concernant la mine d'El Teniente, de se baser sur des résultats de mesure provenant d'essais au vérin effectués sur différents types de laves de la Cordillère des Andes.

Pour le massif d'andésite cisaillée, ont donc été retenues les valeurs suivantes:

- module de déformation : $\mathrm{E}=17000 \mathrm{MPa}$;

- coefficient de Poisson: $v=0,25$.

\section{3}

\section{Estimation du champ de contraintes géostatiques autour de la galerie}

Nous avons vu au paragraphe 2 que la connaissance du champ des contraintes géostatiques au voisinage des galeries était fondamentale pour la modélisation.

Plusieurs mesures de contraintes effectuées dans des endroits divers du niveau Sub 6 étaient disponibles (les mesures effectuées par surcarottage).

Une analyse détaillée de ces mesures a été effectuée, en vue d'estimer les champs de contraintes autour des galeries et leurs variations avec leur position par rapport au front de foudroyage, puisque c'est en effet à l'approche du front de foudroyage qu'il faut s'attendre aux plus fortes variations (voir \$2).
Pour le modèle, dans lequel était simulée la pose des différents éléments de soutènement, il était toutefois essentiel de pouvoir disposer d'une estimation aussi fiable que possible du champ des contraintes géostatiques prévalant à l'excavation de la galerie. C'est en effet uniquement de cette manière que pouvaient être calculés les efforts repris par ces éléments de soutènement juste après leur mise en place.

Il a été décidé d'adopter, pour décrire le champ des contraintes géostatiques initial (c'est-à-dire avant excavation des galeries) au niveau Sub 6, les résultats d'une autre étude, réalisée par um cabinet d'ingénierie américain, portant sur l'analyse des contraintes à l'échelle de toute la mine et du massif rocheux alentour. Ce champ de contrainte, projeté sur le plan du modèle, est représenté sur la figure 3 . Il est intéressant de remarquer que les contraintes horizontales y sont plus élevées que les contraintes verticales.

Il était ensuite nécessaire, pour se replacer dans la situation de la grande majorité des rockbursts observés (en particulier pour le rockburst du 23 mai 1991), d'estimer le champ des contraintes modifié à l'approche du front de foudroyage (à une centaine de mètres en avant de ce front, voir \$2).

Celles des mesures de contraintes in situ effectuées à l'approche du front de foudroyage donnaient des résultats très dispersés, les contraintes majeures principales variant de 30 à $120 \mathrm{MPa}$ ! Néanmoins, sur une vue en plan, apparaissaient clairement deux caractéristiques communes à la majorité de ces mesures : une forte ovalisation du champ de contrainte, et une orientation de la contrainte principale majeure suivant une direction parallèle au plan du front de foudroyage.

Pour représenter l'état de contrainte autour des galeries à l'approche du front de foudroyage, il a donc été décidé de sélectionner celle des mesures qui paraissait la plus représentative pour le rockburst du 23 mai 1991, tout en ajustant les valeurs de manière que la contrainte principale majeure soit égale à la valeur moyenne de $50 \mathrm{MPa}$, estimée par l'étude américaine déjà évoquée plus haut.

Le champ de contrainte correspondant est représenté sur la figure 3.

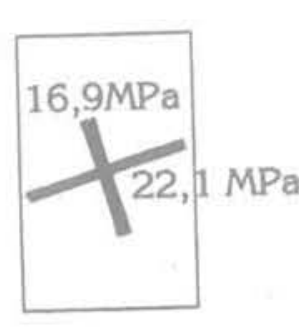

(a)

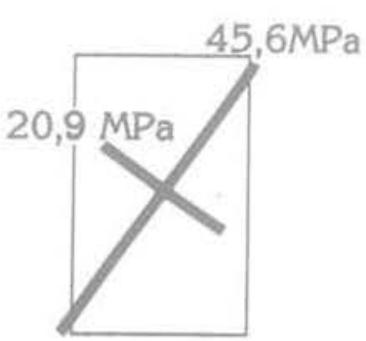

(b)
ค16.3 Champ des contraintes géostatiques projeté dans le plan du modèle (a) avant excavation des galeries du niveau Sub 6 ,

(b) à l'approche du front de foudroyage. In situ stress field projected in the plane of the model mesh

(a) before excavation of galleries in the Sub 6 level,

(b) as the caving front nears by. 
L'approche du front de foudroyage sera donc simulée par le passage du premier au second champ de contraintes, les phénomènes de rockburst intervenant, pour la grande majorité d'entre eux, dans cette dernière situation.

\section{4}

\section{Méthode de modélisation des éléments de soutènement de la galerie}

Le soutènement installé systématiquement dans les galeries du niveau de ventilation est constitué d'ancrage à scellement continu associés à du béton projeté armé de treillis soudé. Les ancrages sont disposés de manière asymétrique, pour tenir compte des caractéristiques du champ de contrainte géostatique (voir figure 2).

Les caractéristiques de l'acier constituant les ancrages sont connues, la limite d'élasticité est de $280 \mathrm{MPa}$ et la contrainte nominale à la rupture est donnée égale à $440 \mathrm{MPa}$.

Des essais effectués en laboratoire sur les mêmes ancrages scellés dans un bloc de béton, ont permis de déterminer un allongement moyen à la rupture de $17 \%$. Par conséquent, les ancrages à scellement continu ont été représentés dans le modèle par des éléments-poutres obéissant à la loi de comportement élastoplastique correspondante, représentée sur la figure 4.

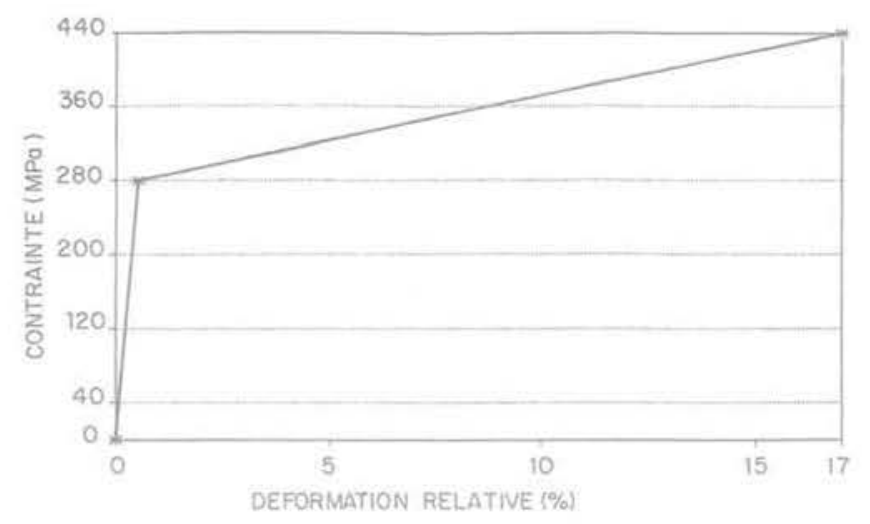

FlQ. 4 Diagramme contrainte-déformation des barres d'ancrages.

Stress-deformation graph of the anchors.

Malheureusement, la mine ne disposait d'aucuns résultats d'essai d'arrachement in situ permettant de vérifier ces hypothèses.

Bien entendu, et afin de ne pas introduire de discontinuités artificielles dans le massif rocheux, des éléments-joints au plan parallèle à celui du modèle ont été introduits entre chacun des ancrages et la roche. Ces éléments-joints obéissaient à une loi de frottement du type Mohr-Coulomb reproduisant autant que possible, en l'absence de données précises, un glissement entre acier et coulis de scellement.

Le béton projeté armé de treillis soudé, dont les caractéristiques étaient connues, a été introduit sous forme d'éléments-poutres sur le parement de la galerie (excepté en radier, lequel n'est pas renforcé en partie courante des galeries).

\section{5}

\section{Analyse statique préliminaire}

\section{1 \\ Les différentes phases de calcul}

Avant la simulation du rockburst lui-même, il est nécessaire de mener une analyse statique préliminaire reproduisant l'historique de la galerie jusqu'à l'approche du front de foudroyage.

La partie statique de la simulation comportera par conséquent quatre étapes, qui sont les suivantes :

- application sur le modèle du champ des contraintes géostatiques initial;

- excayation des galeries :

- mise en place du soutènement ;

- modification du champ de contraintes à l'approche du front de foudroyage.

\section{2}

\section{Excavation et pose du soutènement}

Il a été supposé ici que le soutènement (ancrages à scellement continu et béton projeté associé à du treillis soudé) était mis en place assez loin du front ; c'est en effet ce qui se passe dans la réalité, puisque c'est seulement après avoir excavé une certaine longueur de galerie que le soutènement est mis en œuvre.

Les résultats du calcul après excavation et activation des éléments de soutènements sont assez classiques : la convergence de la galerie reste faible (au plus de l'ordre du centimètre), tandis que les contraintes dans les ancrages à scellement continu tournent autour de $30 \mathrm{MPa}$ pour les plus élevées (à la tête des ancrages).

\section{3}

\section{Approche du front de foudroyage}

A l'approche du front de foudroyage, le modèle se déforme sous l'effet de la brusque modification du champ des contraintes géostatiques, comme représenté sur la figure 5 .

L'allure de la déformation de la galerie ne surprend pas, car elle correspond au schéma classiquement observé dans la mine.

Plus surprenante, et bien mise en évidence par la modélisation, est l'ampleur des déplacements correspondants à l'échelle du massif rocheux entourant la galerie (déplacement subhorizontal de près de $10 \mathrm{~cm}$ pour un bloc de $55 \mathrm{~m}$ de hauteur).

Ce déplacement est bien évidemment lié à la taille et à la configuration du modèle choisi, mais il donne une idée des très fortes variations de contraintes et de l'ampleur des déformations qui résultent de l'approche du front de foudroyage.

Il est intéressant de faire remarquer que ce résultat n'a pas surpris les responsables de la mine, dans la mesure où des déplacements de l'ordre de la dizaine de centimètres ont effectivement pu être mesurés en topographie dans les galeries après passage du front de foudroyage.

Parallèlement, une plastification importante du massif rocheux autour de la galerie est observée, avec, dans 


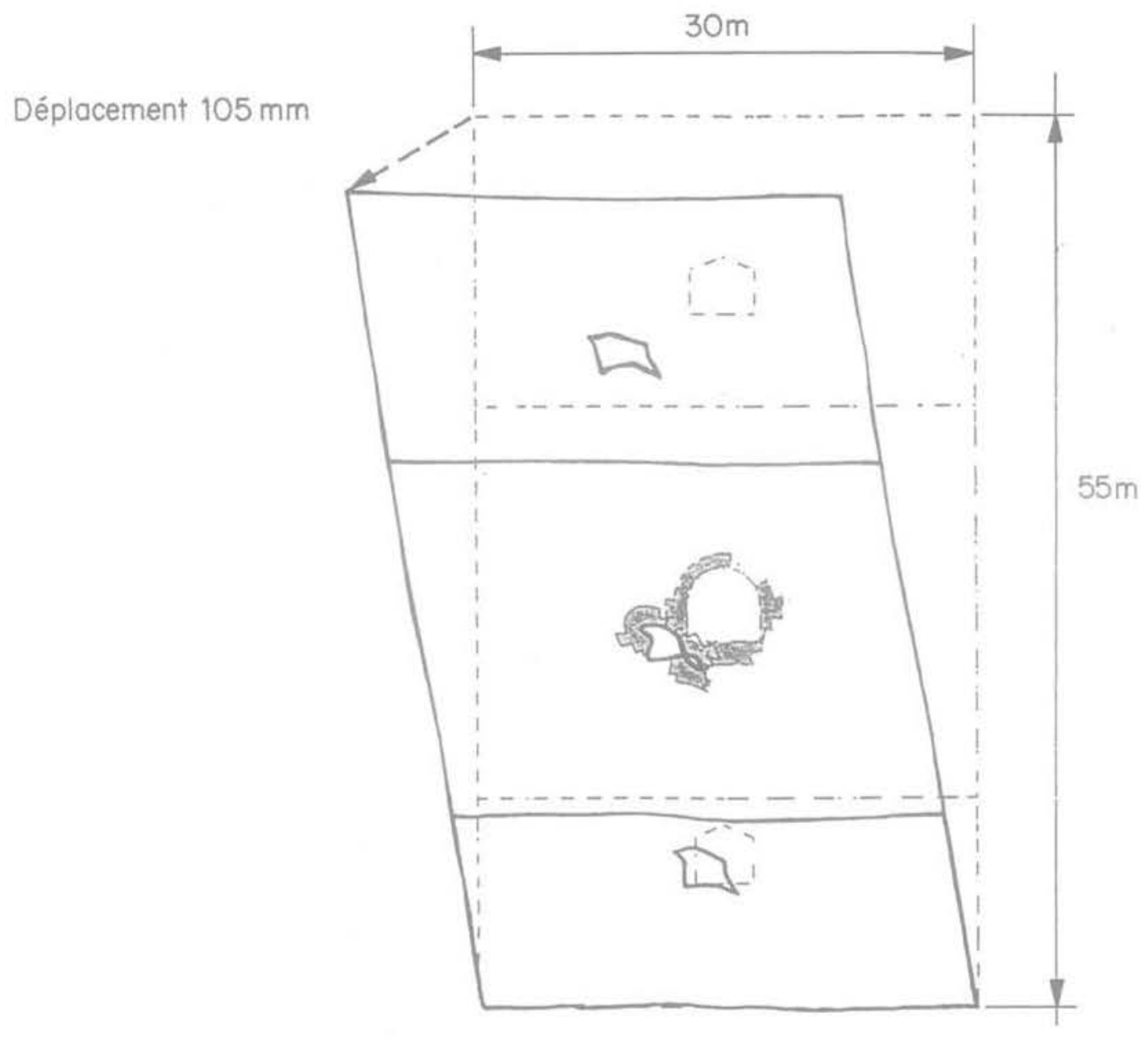

Roche décomprimée au voisinoge de la galerie

Déplacements à l'échelle du modèle sous l'effet de la modification du champ des contraintes à l'approche du front de foudroyage (échelle $1 / 400$, déplacements multipliés par 80 ).

Displacements of the model mesh as calculated for the modified stress field, as the caving front nears by (scale 1/400, displacements exaggerated by a factor of 80 )

ces zones, des contraintes dans les éléments d'ancrage dépassant la limite d'élasticité de l'acier (280 MPa), comme visible sur la figure 6 . Ce résultat n'a pas non plus surpris les exploitants de la mine pour lesquels le soutènement est effectivement extrêmement sollicité, jusqu'à la plastification.

Les contraintes de compression dans les éléments de béton projeté armé font apparaître de leur côté que la coque de béton projeté n'est plus en mesure de jouer un rôle de soutènement au passage du front de foudroyage. Dans la pratique, elle sert toutefois à empêcher les chutes de blocs ou de masses de rocher broyé dans les galeries.

Les déplacements le long des joints entre ancrages et massif rocheux restent par contre négligeables, ce qui montre que les ancrages suivent pratiquement les mouvements du rocher encaissant.

\section{4}

\section{Conclusions de l'analyse statique}

L'analyse statique qui précède a confirmé la très forte influence du champ de contraintes autour de la galerie sur son comportement et sur les éléments de son soutènement. Il apparaît en effet que l'approche du front de foudroyage s'accompagne de fortes déformations, et d'une plastification du rocher et de certaines sections d'ancrages parmi les plus sollicitées (dans les zones plastifiées).

De plus, l'analyse statique met en évidence de manière très claire le fait que le massif rocheux à l'avant du front de foudroyage, soumis à une modification brutale du champ de contraintes, doit théoriquement, pour obéir à ces nouvelles contraintes, se déformer de façon non négligeable (dans le cas de notre modèle, presque $10 \mathrm{~cm}$ de déplacement subhorizontal pour un bloc de $55 \mathrm{~m}$ de hauteur).

Devant l'ampleur de ces déformations, il n'est par conséquent pas étonnant que le massif rocheux subisse des réajustements aux alentours des zones foudroyées, lesquels réajustements se font probablement par le rejeu de discontinuités géologiques existantes pour lesquelles l'état d'équilibre n'est plus respecté avec le nouveau champ de contraintes. C'est bien l'interprétation principale qui est donnée des phénomènes de rockbursts dans la mine d'El Teniente, qui seraient donc des événements sismiques provoqués par le rejeu de dis- 


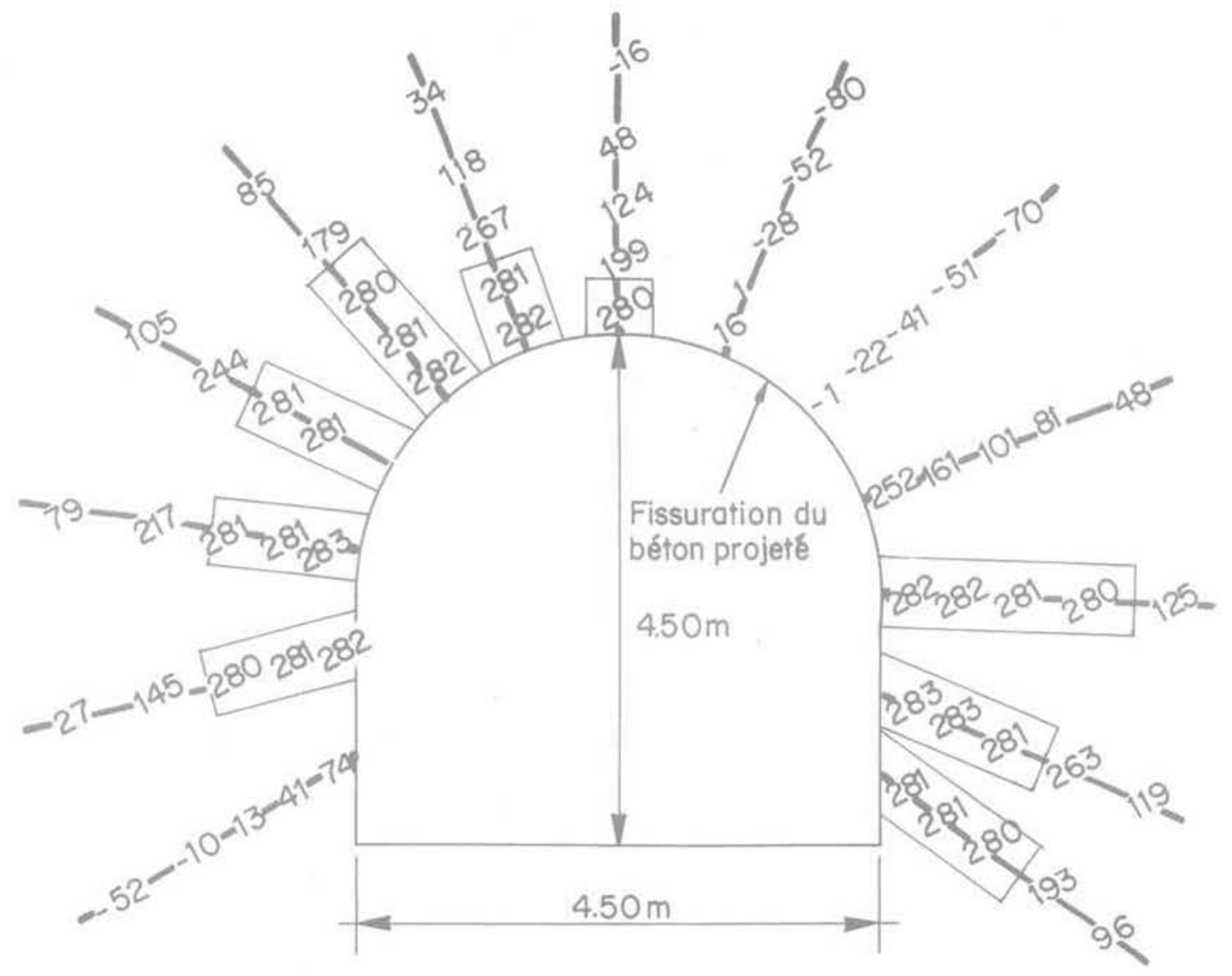

$\square$ Longueur d'ancrages dépassant la limite d'élasticité de l'acier.

AG.6 Contraintes (en MPa) dans les ancrages à scellement continu à l'approche du front de foudroyage (les valeurs négatives correspondant à des compressions).

Stresses (in MPa) within the grouted anchors, as the caving front nears by (a negative value correspond to a compression).

continuités au voisinage immédiat des zones foudroyées (le rockburst du 23 mai 1991 a d'ailleurs bien été détecté comme une secousse sismique, comme mentionné au paragraphe 2).

Dans le cadre de cette hypothèse qui apparaît très vraisemblablement au vu des résultats obtenus, l'effet des rockbursts sur les galeries de mine serait en fait équivalent à celui d'un séisme dont le foyer ne se situerait qu'à quelques centaines de mètres au plus.

\section{6}

\section{Analyse dynamique de simulation du rockburst}

\section{1}

\section{Les données disponibles et le mode de simulation du rockburst}

Le rockburst a été simulé par l'application sur le modèle d'ondes de choc. Afin de tenir compte de la proximité de la source sismique, les sollicitations dynamiques ont été supposées se propager dans le modèle comme une onde plane, plutôt que comme une onde sphérique.

Le principal obstacle rencontré dans cette modélisation a toutefois été le manque de données concernant le rockburst du 23 mai 1991, que l'on cherchait à reproduire ici, et les phénomènes de rockburst à El Teniente en général.

En effet, si actuellement existe au niveau Sub 6 de la mine un dispositif complet d'acquisition des données permettant de recueillir les accélérogrammes mesurés en différents points, ce dispositif n'existait pas encore lorsque cette étude a été entamée. Les seules données disponibles consistaient alors en trois enregistrements de rockbursts effectués lors d'essais de ce dispositif, dont l'un enregistré pour un événement de magnitude 1.

Concernant le rockburst du 23 mai 1991, la seule donnée disponible était sa magnitude, alors estimée à 3 .

Il a donc été nécessaire de construire un accélérogramme de rockburst sur la base de l'enregistrement des vitesses particulaires en fonction du temps durant l'événement de magnitude 1 dont nous disposions, les amplitudes et les fréquences de cet enregistrement 
étant modifiées de manière à obtenir une magnitude de 3.

Les variations de la composante horizontale de la vitesse pour le signal retenu sont représentées sur la figure 7 ; la vitesse particulaire maximale étant d'environ $10 \mathrm{~cm} / \mathrm{s}$.

Le spectre d'accélération correspondant montre de très hautes fréquences, essentiellement entre 100 et $300 \mathrm{~Hz}$. De telles valeurs ne sont cependant pas surprenantes étant donnée la proximité du fover, et sont souvent caractéristiques des événements de rockbursts.

\section{9.}

\section{Descriptions des résultats obtenus}

Pour l'analyse dynamique, seule la galerie centrale a été modélisée, les deux autres galeries incorporées dans le modèle statique, et représentant les niveaux inférieur et supérieur, n'étant pas apparues apporter une influence notable aux contraintes et déformations au voisinage de la galerie du niveau de ventilation considérée.

Le maillage a été conçu dès le départ suffisamment fin pour permettre de reproduire les effets d'ondes de fréquences élevées, comme celles contenues dans le spectre utilisé. De plus, des éléments numériques placés sur tout le pourtour du modèle assurent l'absorption des ondes sismiques et évitent leur réflexion sur les bords du modèle.

Le calcul dynamique élastoplastique a été exécuté avec un pas de temps de 0,1 milliseconde.

Les résultats, sauvegardés toutes les dix étapes, c'est-à-dire toutes les millisecondes, ont permis de visualiser à tout instant les champs de contraintes, les déformations et les efforts dans les éléments de soutènement. La vitesse particulaire maximale atteinte lors de la simulation sur le parement de la galerie est de $13 \mathrm{~cm} / \mathrm{s}$; elle est atteinte au passage sur le modèle du pic de vitesse visible sur la figure 7 .

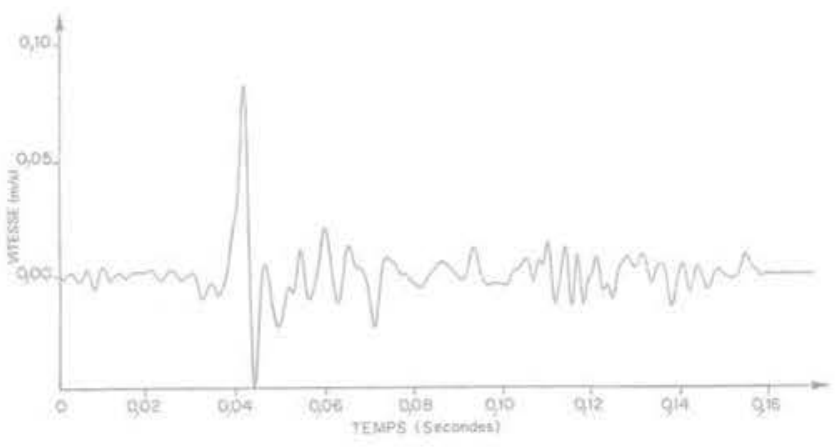

FG. T Diagramme de vitesse en fonction du temps utilisé pour la simulation du rockburst du 23 mai 1991 (composante horizontale).

Evolution of the velocity versus time for simulation of the May 23. 1991 rockburst (horizontal component)

Les déplacements du parement de la galerie durant la simulation n'excèdent pas quelques dixièmes de millimètres, tandis que les contraintes de traction dans les ancrages restent pratiquement inchangées.
Une animation vidéo a été réalisée, montrant les déformations de la galerie sous l'effet des ondes sismiques. La figure 8 est une photo extraite de cette vidéo, et représente la déformation de la galerie au pic de vitesse.

Les déformations qui subsistent en piédroit gauche et en radier de la galerie après le passage du train d'ondes sismiques montrent bien le comportement élastoplastique du rocher dans ces zones, déjà reconnues comme plastifiées dans le calcul statique, à l'approche du front de foudroyage. Néanmoins, il est évident que ces résultats ne pouvaient pas expliquer à eux seuls les graves dommages subis par les galeries lors des phénomènes de rockbursts

Une réflexion a donc été menée afin d'essayer de comprendre pourquoi, malgré l'attention apportée aux choix des données, les résultats ne reflètent pas le caractère extrêmement dommageable des rockbursts.

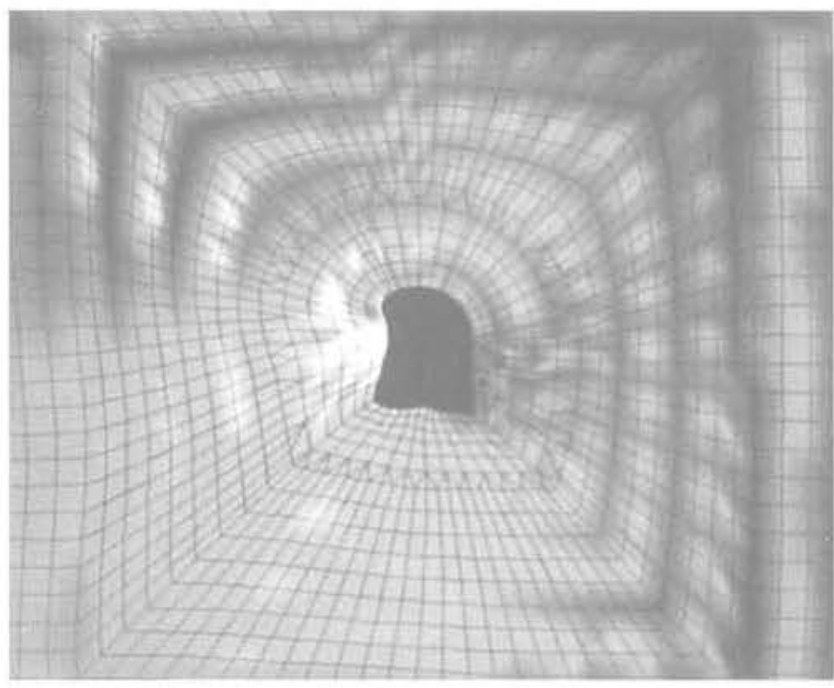

คด. 8 Déformẻe de la galerie au passage du pic de vitesse, durant la simulation du rockburst: les déplacements sont multipliés par 50 (photo extraite de l'animation vidéo réalisée à partir des résultats du calcul).

Deformation of the gallery under peak velocity during simulation of the rockburst

displacements are exaggerated by a factor of 50 (photo from the video film which was realized from the calculation results).

\section{Améliorations envisageables pour la simulation de phénomènes de rockbursts}

Il est certain que les données sur les événements de rockbursts au moment où la présente étude a été initiée, étaient très réduites. En particulier, il aurait fallu pouvoir disposer d'enregistrements directement à proximité des galeries affectées par le rockburst du 23 mai 1991. dont on a cherché à reproduire les conditions par la présente modélisation.

Il apparaît nécessaire, pour une bonne compréhension du phénomène, de disposer de nombreuses données en des points très divers, afin d'une part de carac- 
tériser de manière précise les sollicitations sismiques auxquelles les galeries sont susceptibles d'être soumises, et d'autre part d'essayer d'identifier le mécanisme à la source.

Dans le cas de notre simulation, il est naturel de se poser la question de savoir si les données utilisées étaient réellement représentatives. Notons, au passage, que la magnitude du rockburst du 23 mai 1991 a été depuis révisée à la hausse : elle est estimée comprise entre 3,9 et 4,1 .

Il a été vu dans l'analyse statique préliminaire, simulant l'approche du front de foudroyage, que la galerie et ses éléments de soutènement étaient extrêmement sensibles à toute modification du champ des contraintes dans le massif encaissant. Or, un rockburst, en tant qu'événement sismique survenant à proximité directe des galeries, provoque lui aussi une relaxation des contraintes et un réajustement du massif autour du foyer.

Le foyer lui-même, vue sa proximité, ne peut être réduit à un simple point dans l'espace : s'agissant d'un mouvement le long d'une ou de plusieurs discontinuités, il est plus exact de parler de «zone-source ». En conséquence, la (c zone-source ») étant située directement aux abords des galeries, il apparait, après coup. que l'effet d'un rockburst proche ne peut pas être simulé correctement uniquement par l'application d'ondes sismiques. L'événement s'accompagne nécessairement d'une redistribution des contraintes dans la " zone-source » et partout aux alentours.

Une simulation devrait donc pouvoir tenir compte de la modification du champ des contraintes géostatiques qui accompagne le brusque relâchement de contraintes causant la secousse sismique.

\section{4}

\section{Réflexions sur l'adéquation des moyens de soutènement mis en œuvre}

La figure 6 montre la distribution des contraintes dans les divers éléments des ancrages à scellement continu utilisés dans les galeries, à l'approche du front de foudroyage. Les ancrages apparaissent ainsi sollicités au-delà de leur limite élastique, tout spécialement dans les zones plastifiées autour de la galerie.

De plus, la résistance à la compression du béton projeté est dépassée pour la quasi-totalité des éléments, ce qui correspond bien à la fissuration extrême observée dans les galeries.

Afin de distribuer les contraintes de traction de manière plus uniforme le long des ancrages, il a paru intéressant de suggérer l'utilisation d'ancrages à scellement ponctuel (ancrages à coquilles). La figure 9 montre la valeur des contraintes dans des ancrages à scellement ponctuel (supposés de longueur identique à ceux actuellement employés) dans le cas de l'approche du front de foudroyage. Les contraintes apparaissent beaucoup mieux réparties avec, toutefois, quatre ancrages atteignant juste la limite d'élasticité,

Les recommandations quant aux moyens de soutènement à mettre en œuvre pour assurer une meilleure tenue des galeries aux rockbursts (et donc aussi vis-àvis des modifications du champ de contraintes lors des foudroyages) ont donc été dans le sens d'une plus grande souplesse du soutènement, permettant autant

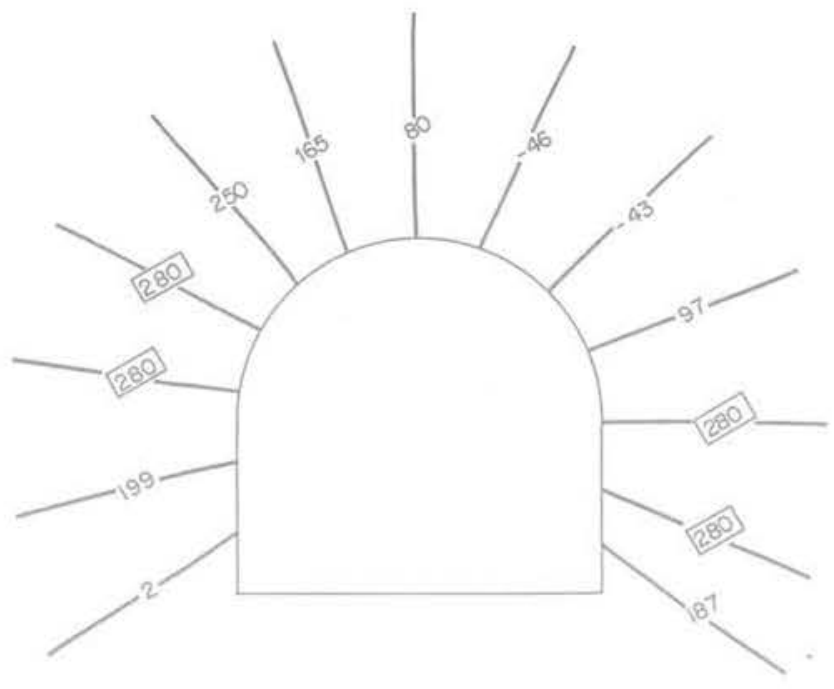

FG.9. Contraintes (en mégapascals) obtenues par le calcul dans le cas de l'utilisation de boulons à ancrage ponctuel de même longueur que les ancrages à scellement continu actuellement utilisés (limite élastique $280 \mathrm{MPa}$, les valeurs négatives correspondent à des compressions). Stresses (in megapascals) obtained from the calculation in case of using non-grouted rockbolts of same length than the actually used grouted anchors (yield stress $280 \mathrm{MPa}$, negative values correspond to a compression).

que possible d'absorber de grandes déformations sans pour autant menacer la stabilité de l'excavation.

Dans cet esprit, il a été recommandé de réaliser des sections d'essai soutenues au moyen d'ancrages ponctuels de grande longueur (de manière à répartir les contraintes jusqu'aussi loin que possible à l'intérieur du massif), solidarisés avec un grillage suffisamment robuste et une coque de béton projeté.

Enfin, l'extension systématique du soutènement en radier a été très fortement recommandée, de nombreuses galeries s'étant trouvées, lors de rockbursts, gravement endommagées par soulèvement de leur radier. Les calculs montraient également l'amélioration possible de la stabilité par l'application d'une pression de confinement sur le radier.

\section{Conclusions}

En dehors des problèmes de données concernant les rockbursts à El Teniente, la présente étude a permis de montrer qu'une modélisation correcte d'une galerie et de ses éléments de soutènements soumis à des ondes sismiques pouvait être menée (dans le cas présent, grâce au code de calcul aux éléments finis GEFDYN). Les effets des différentes phases de l'exploitation minière ont également pu être simulés de manière très satisfaisante.

Cette étude constituant, semble-t-il, la première tentative de simulation des effets d'un rockburst sur une galerie de mine et son soutènement, il est clair que le degré de complexité du modèle a dû être limité, d'où 
des approximations inévitables, dont notamment le choix d'un modèle bidimensionnel.

Outre les hypothèses qui ont dû être faites à cause de certaines lacunes de données, les foyers des événements sismiques qui, très probablement causent les rockbursts, sont trop proches des galeries, de telle sorte qu'un rockburst ne peut être réduit à la simple propagation d'ondes sismiques.

C'est ainsi qu'en plus de l'écoute sismique, l'évolution des champs de contraintes autour des galeries devrait être suivie de manière fiable et régulière pour une bonne définition des données qui seraient à introduire. Ceci permettrait notamment d'étudier de manière précise les variations du champ de contraintes lors de phénomènes de rockbursts.

Une meilleure connaissance dans les mécanismes de génération des rockbursts à El Teniente est aussi nécessaire pour définir le cadre d'un modèle amélioré de simulation de rockburst. C'est pourquoi la mine d'El Teniente fait actuellement un gros effort dans l'acquisition des données nécessaires.

Bénéficiant des données supplémentaires qui ne manqueront pas d'être recueillies et de l'expérience acquise lors de cette étude, on peut songer à améliorer de manière substantielle la modélisation, par exemple en incorporant la source sismique dans le modèle, qui pourrait lui-même, dans un stade ultérieur être tridimensionnel.

Enfin, il ne faut pas négliger les conclusions très importantes que le modèle a permis de mettre en évidence concernant les moyens de soutènement. Ces conclusions se sont d'ailleurs trouvées correspondre à celles déjà formulées sur la base de l'expérience.

La réalisation de sections d'essai au niveau Sub 6 de la mine, comportant divers types de soutènement, devrait apporter, de ce point de vue des indications précieuses, et permettre l'exploitation dans de meilleures conditions de sécurité.

\section{NOTA}

La suite de cette étude a été soutenue par le fonds de la Recherche et de la Technologie du ministère francais de la Recherche, dans le cadre du saut technologique ARSINOE $3+2$.

\section{$\overline{\text { Bibliographie }}$}

Bouvard-Lecoanet A, Colombet G, Esteulle F, - Ouvrages souterrains: conception, réalisation, entretien, 2 éd. Presses des Ponts et Chaussées, 1992. Corbetta F., Bernaud D., Nquyen Minh D. - Contribution à la méthode convergence-confinement par le principe de la similitude, Revue Française de Géotechnique, $n^{\circ}$ 54, 1991, pp. 5-11.

Hoek E., Brown E.T. - The Hoek-Brown failure criterion : a 1988 update. Proceedings of the 15th Canadian Rock Mechanics Symposium, Toronto, 1988.
McGarr A., Green R.W.E., Spottiswoode S.M. - Strong ground motion of mine tremors : some implications for near-source ground motion parameters, Bull of the Seismological Society of América, vol. $71, n^{\circ} 1,1981, \mathrm{pp}, 295$. 319. 